\title{
Journal of

\section{Morphology control via dual solvent crystallization for high-mobility functionalized pentacene-blend thin film transistors $\dagger$}

\author{
Maria Lada, ${ }^{* a}$ Marco J. Starink, ${ }^{a}$ Miguel Carrasco, ${ }^{b}$ Lichun Chen, ${ }^{b}$ Pawel Miskiewicz, ${ }^{b}$ Paul Brookes, ${ }^{b}$ \\ Margaret Obarowska ${ }^{c}$ and David C. Smith ${ }^{c}$
}

Received 15th March 2011, Accepted 9th May 2011

DOI: 10.1039/c1jm11119a

\begin{abstract}
We present an approach to improving the performance of solution processed organic semiconductor transistors based on a dual solvent system. We here apply this to a blend containing the $\pi$-conjugated small molecule 6,13 bis(triisopropylsilylethynyl) pentacene (TIPS-pentacene) and polystyrene, which acts as an inert binder. Using a semiconductor-binder solution of two solvents, where the main solvent is a better solvent of the small molecule and second solvent is a better solvent of the polymer, crystal morphologies can be altered and transistor mobilities increased by almost an order of magnitude. In this way, air-ambient and solution-processed transistors with linear and saturation mobilities higher than $1 \mathrm{~cm}^{2} \mathrm{~V}^{-1} \mathrm{~s}^{-1}$ have been fabricated. We discuss how the solubility properties of the formulation components can be used to identify solvent candidates that promote an efficient self-assembly of the small molecule.
\end{abstract}

\section{Introduction}

In the search for high performance organic semiconductor (OSC) materials, control and cost of device processing steps are critical. For this reason, solution-based processing techniques have emerged as the most promising in terms of fulfilling production requirements for the realization of low-cost, high functionality electronics, such as, for example, thin film transistor circuits (TFTs) for flexible active matrix backplanes. ${ }^{1}$ Thus far, soluble acene-based small molecules stand out as fulfilling many production requirements including high mobilities. Among those, 6,13-bis(triisopropylsilylethynyl) pentacene (TIPS-pentacene) is popular as it combines good charge transfer characteristics with high solubility. ${ }^{2}$ Nevertheless, achieving optimum film morphology remains a challenge and high mobilities $\left(>1 \mathrm{~cm}^{2} \mathrm{~V}^{-1}\right.$ $\mathrm{s}^{-1}$ for small molecules and $\sim 0.1 \mathrm{~cm}^{2} \mathrm{~V}^{-1} \mathrm{~s}^{-1}$ for polymers) mostly rely on single crystal techniques and very careful in situ control of the crystallization process. ${ }^{3-5}$ However, such techniques are demanding and costly, and are limited due to a narrow range of processing conditions.

To overcome such difficulties, a formulation approach has been proposed by which a polymeric binder is added into the solution. ${ }^{6}$ At deposition-performed for example by spin

${ }^{a}$ School of Engineering Sciences, University of Southampton, University Road, Southampton,SO171BJ, UK.E-mail: ladas@technai.com

${ }^{b}$ Merck Chemicals Ltd., Chilworth Technical Centre, University Parkway, Southampton, SO16 7QD, UK

'School of Physics, University of Southampton, University Road, Southampton, SO17 1BJ, UK

$\dagger$ Electronic supplementary information (ESI) available. See DOI: $10.1039 / \mathrm{c} 1 \mathrm{jm} 11119 \mathrm{a}$ coating - the polymer contained in the blend might influence the deposition in several ways, for instance it may prolong the drying time and change the diffusion kinetics in the drying layer. This may allow for better self-organization of the small molecule and, consequently, enhanced device performance. Produced films benefit from the superior structural properties of the polymer, showing absence of cracking, improved substrate wetting and high film thickness uniformity. In addition, the tunable rheological properties of the solution open the way for other low-cost deposition processes such as printing. The blend approach, therefore, allows for high functional performance offered by the small molecule combined by good structural performance provided by the polymer. High mobilities of the resultant transistors are due to a spontaneous phase separation by which the small molecule is segregated towards one or both interfaces of the film, ${ }^{7,8}$ depending on the energetics of the blend components and the surface energy of the substrate. In addition, it has been verified that phase separation can be enhanced by post-deposition anneal close to the glass-transition temperature of the polymer. ${ }^{9}$ However, since the small-molecule is thought to be diluted to some extent into the binder there has been considerable debate about a potentially limiting role of the binder in the resultant charge mobility. ${ }^{10}$

To date, the maximum device mobility of a TIPS-pentacene blend TFT reported in the published literature has been for the OSC binder, polytriarylamine $\left(1.1 \mathrm{~cm}^{2} \mathrm{~V}^{-1} \mathrm{~s}^{-1}\right) .{ }^{8}$ Blends of TIPSpentacene with inert binders have yielded mobilities only up to $0.54 \mathrm{~cm}^{2} \mathrm{~V}^{-1} \mathrm{~s}^{-1}$ (bottom gate) ${ }^{9}$ and $0.69 \mathrm{~cm}^{2} \mathrm{~V}^{-1} \mathrm{~s}^{-1}$ (top gate) $)^{8}$ $\operatorname{poly}(\alpha$-methyl styrene) was used in both cases. These results were reported for devices annealed in a nitrogen atmosphere. When devices were processed in air, mobilities were much lower, 
typically of the order of or less than $\sim 0.1 \mathrm{~cm}^{2} \mathrm{~V}^{-1} \mathrm{~s}^{-1}$, both for bottom and top gate devices. ${ }^{8,11,12}$

So far, some reports have concluded that when inert binders such as amorphous polystyrenes are used in semiconducting blends, lower performance of devices is an inherent result of their non-crystalline structure. ${ }^{11}$ It has, therefore, been conjectured that in this case crystalline-crystalline phase would offer better control over phase separation and better device performance potentially due to a better control of which phase would crystallize first. ${ }^{10}$

Another approach in improving self-assembly of the functional molecule is to better control diffusion in the drying film by reducing the evaporation rate using, for example, a higher boiling point solvent. ${ }^{13}$ Environmentally friendly solvents with high acene solubility can be found in the group of alkyl aromatics, such as toluene, xylene, etc. We have found that in this way mobilities close to but not much higher than $1 \mathrm{~cm}^{2} \mathrm{~V}^{-1}$ $\mathrm{s}^{-1}$ could be achieved. The appearance of such a limit is thought to be due to the benefit of a higher boiling point solvent being counterbalanced by reduced solubility for both solutes.

In the present work, we report on a novel approach to further increase mobilities, in which a second, more polar solvent is added to the main solvent. This is thought to be potentially beneficial as it allows enhanced crystallization of the small molecule but at the same time increases the solubility of the polymer.

\section{Results and analysis}

\subsection{Matrix of experiments}

The direct aim of the experiments in this work was to investigate the effect on TFT device performance of two parameters: type of second solvent and TIPS-pentacene:PS ratio which is the mixing ratio by volume of stock solutions $\left(R_{\mathrm{T}-\mathrm{P}}\right)$. The stock solutions were prepared in $2 \%$ weight content of each solute into the respective solvent. All stock solutions of TIPS-pentacene were prepared in mesitylene-designated as the main solvent throughout the experiments. Stock solutions of PS were prepared in a series of second solvents which, apart from mesitylene, included two other alkyl aromatics (cumene and indane), two aromatic acetates (butyl and amyl acetate) and two aromatic ethers (anisole and 4-methylanisole). The whole solvent group was chosen specifically so as to span a range of boiling points around that of mesitylene.

The matrix of experiments consisted of all permutations of the 6 secondary solvents and $R_{\mathrm{T}-\mathrm{P}}=1$ and $R_{\mathrm{T}-\mathrm{P}}=3$. To limit possible effects of lab variability and sample deterioration, care was taken that all experiments were conducted on identical equipment in a time interval that is as short as practically possible. No experiments using a solvent without mesitylene were conducted. This is because polar molecules are not suitable as single solvents; e.g. solubility of TIPS-pentacene in anisole was $\sim 1.5 \% \mathrm{w} / \mathrm{w}$, which is too low.

\subsection{Properties and morphology of $\boldsymbol{R}_{\mathrm{T}-\mathrm{P}}=1$ devices}

A first series of blends was prepared by mixing stock solutions in ratio $1: 1\left(R_{\mathrm{T}-\mathrm{P}}=1\right)$. Fig. 1 shows typical transfer curves of devices deposited from mesitylene and mesitylene-anisole
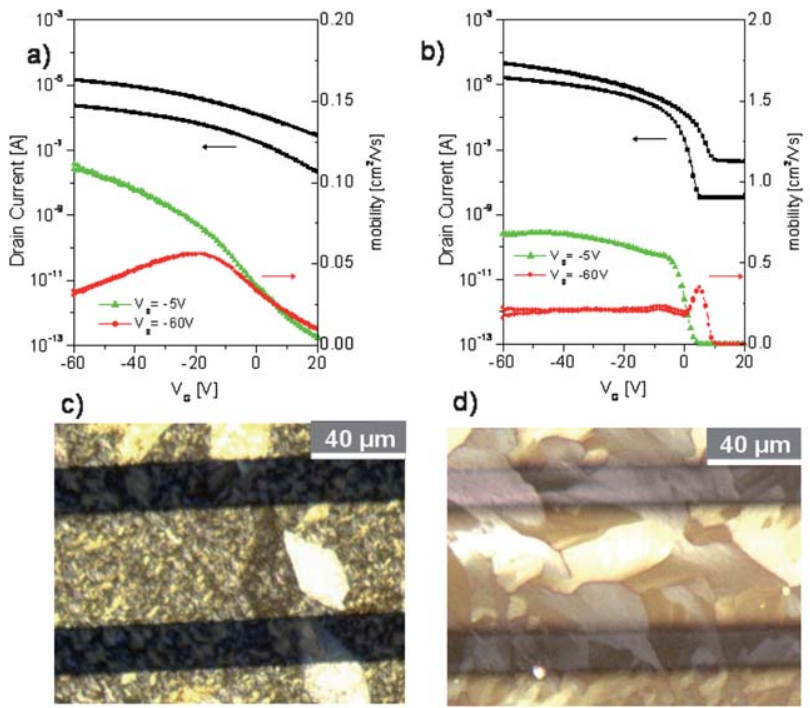

e)
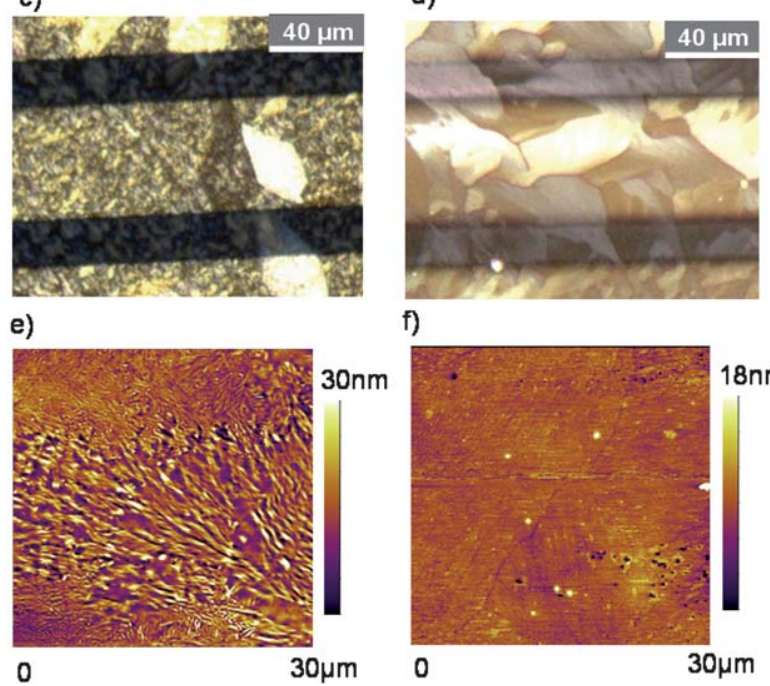

f)

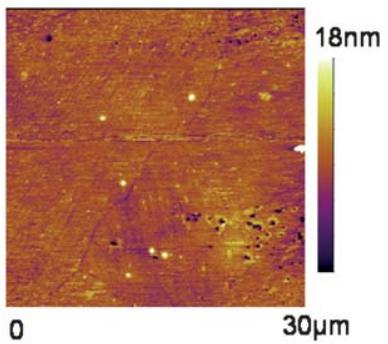

Fig. 1 Performance and morphology for devices based on TIPS-pentacene:PS blends deposited from stock solutions mixed in ratio $R_{\mathrm{T}-\mathrm{R}}=1$ using single (i.e. mesitylene only) and dual solvent formulations. Graphs (a) and (b) are typical transfer characteristics in the linear and saturation regimes, corresponding to single, mesitylene (graph a) and dual, mesitylene-anisole solutions (graph b). Corresponding optical micrographs under crossed polarizers and AFM height scans show resultant crystallite sizes and surface morphologies respectively (single solvent: images (c) and (e); dual solvent: images (d) and (f)). A strong improvement in transistor performance for the dual solvent case is evident. This corresponds to a substantial increase in the size of crystallite domains and decrease in surface roughness.

solutions (Fig. 1a and b) as well as optical micrographs (Fig. 1c and d) and atomic force microscopy (AFM) height images (Fig. 1e and $\mathrm{f}$ ) of the corresponding films taken at the active channel area. As can be seen, devices deposited from a single solvent had mobilities of the order of $0.1 \mathrm{~cm}^{2} \mathrm{~V}^{-1} \mathrm{~s}^{-1}$ which is typical for this solute ratio and single solvent processing. ${ }^{8,11}$ On the other hand, addition of anisole has resulted in a strong improvement in device mobilities with values as high as 1.01 $\mathrm{cm}^{2} \mathrm{~V}^{-1} \mathrm{~s}^{-1}$ (linear) and $1.16 \mathrm{~cm}^{2} \mathrm{~V}^{-1} \mathrm{~s}^{-1}$ (saturation). Direct comparison of the optical micrographs shows that addition of anisole in the solution results in a significant increase in crystallite size. For this dual solvent, crystallite domains with sizes larger than $20 \mu \mathrm{m}$ are evident by their birefringence behavior under crossed polarizers. On the other hand, for the single solvent film, there is only a small number of large crystallite domains enclosed in a matrix containing small crystallites or amorphous material. Additionally, from the AFM scans, a reduction in surface roughness for the dual solvent film is 
evident. At this imaging scale, the surface of the dual solvent is mostly featureless - with the exception of some small pinholes and grain boundaries - while the single solvent film exhibits elevations that could be interpreted as needle-shaped microcrystallines with strong directional growth. The size of domains especially in the channel would be expected to impact performance not only regarding mobility and off-current but also threshold voltage, $V_{\mathrm{T}}$. The high $V_{\mathrm{T}}$ observed for the single solvent film is consistent with a large number of traps at grain boundaries and amorphous regions.

\subsection{Properties and morphology of $\boldsymbol{R}_{\mathrm{T}-\mathrm{P}}=\mathbf{3}$ devices}

Due to the structural design of the device (top gate, bottom contact), there is a vertical distance between the active interface and drain-source contacts. As the drying blend strongly phase separates along the vertical direction, the semiconducting molecule predominantly segregates at the free surface where it crystallizes. We consider this to be a key condition for a high mobility device. To examine this hypothesis, we increased the loading ratio of TIPS-pentacene by preparing a second series of blends with stock solutions mixed in ratio $3: 1\left(R_{\mathrm{T}-\mathrm{P}}=3\right)$.

Complete statistics from all devices fabricated can be seen in the ESI (Table $\mathrm{S} 1 \dagger$ ). In Fig. 2, we present a graphical comparison of average and maximum saturation mobilities obtained for $R_{\mathrm{T}-\mathrm{P}}=1$ and $R_{\mathrm{T}-\mathrm{P}}=3$ for films deposited from various solvents. Among the various second solvents, anisole and 4-methylanisole gave average saturation mobilities higher than $1 \mathrm{~cm}^{2} \mathrm{~V}^{-1} \mathrm{~s}^{-1}$ and for individual devices as high as $1.82 \mathrm{~cm}^{2} \mathrm{~V}^{-1} \mathrm{~s}^{-1}$. Typical transfer and output behaviour of such a device can be seen in Fig. 3. To our knowledge, TFT device mobilities of this range are of the highest ever demonstrated for TIPS-pentacene. Typical threshold voltage values in the saturation regime for these devices are 5-7 V with onset voltage values $\sim 7 \mathrm{~V}$, on/off ratio is

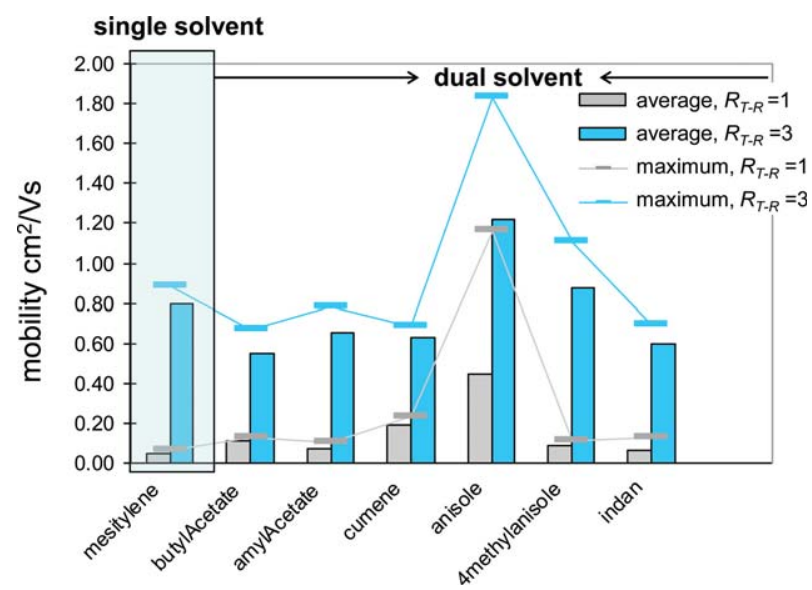

Fig. 2 Saturation mobilities of devices fabricated from single solvent (leftmost bar) and dual solvent solutions of TIPS-pentacene:PS for a series of different second solvents. The second solvents are arranged in ascending boiling point order. Histogram bars show average values and continuous lines show maximum values obtained from 6 random devices. Blue bars and lines correspond to results from stock solutions mixed in ratio $R_{\mathrm{T}-\mathrm{R}}=3$, i.e. higher loading content of TIPS-pentacene, and grey bars and lines to $R_{\mathrm{T}-\mathrm{R}}=1$.
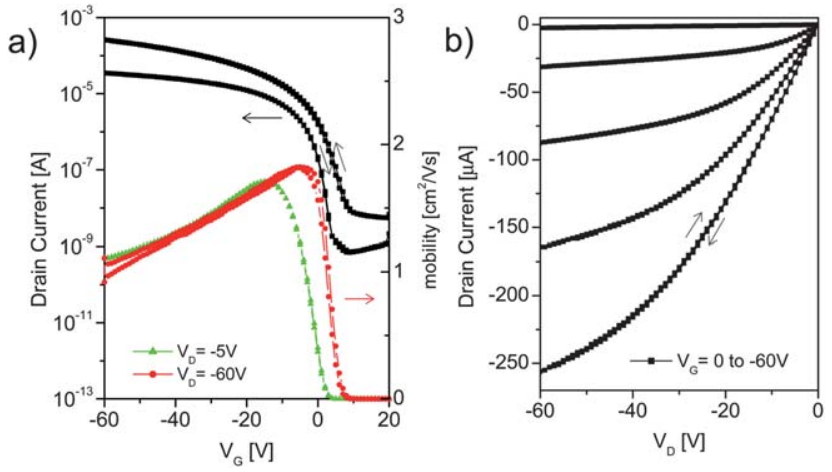

Fig. 3 Typical transfer and output curves of TIPS-pentacene:PS transistor deposited from mesitylene-anisole solution $\left(R_{\mathrm{T}-\mathrm{R}}=3\right)$. Saturation mobility statistics from six random devices gave a mean value of $1.22 \mathrm{~cm}^{2}$ $\mathrm{V}^{-1} \mathrm{~s}^{-1}$ and standard deviation of $0.33 \mathrm{~cm}^{2} \mathrm{~V}^{-1} \mathrm{~s}^{-1}$.

$\sim 10^{4.5}$ and both $V_{\mathrm{G}}$ (transfer) and $V_{\mathrm{D}}$ (output) sweeps (performed in the positive-negative-positive cycle) are indicative of minimal hysteresis. Additionally, air stability measured over a period of one month was excellent with devices maintaining mobility within $5 \%$ of the originally measured value and stable off-current.

The surface morphology of typical layers at the active channel area from single and dual solvents obtained using the mixing ratio $R_{\mathrm{T}-\mathrm{P}}=3$ was further characterized with optical microscopy and AFM. Fig. 4 shows optical images deposited from mesitylene (single solvent) and mesitylene-anisole (dual solvent) a)

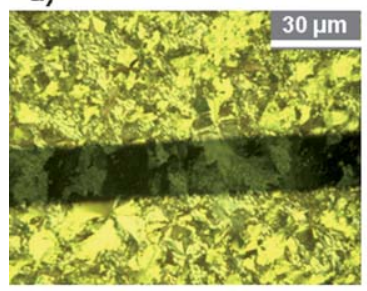

c)

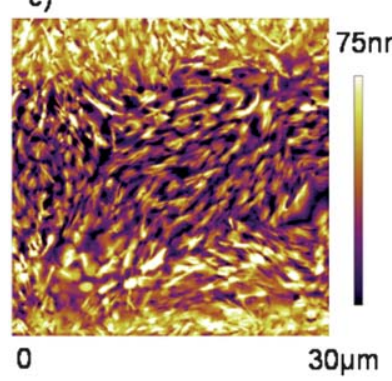

b)

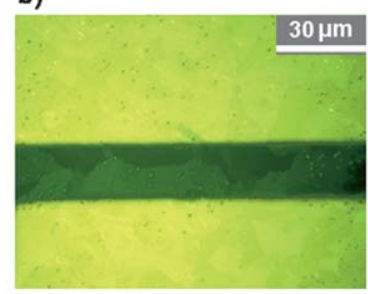

d)

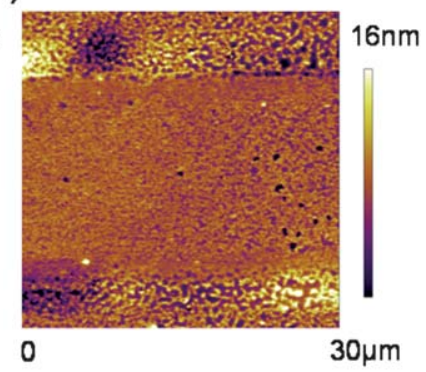

Fig. 4 Structural morphology of TIPS-pentacene:PS films deposited from stock solutions mixed in ratio $R_{\mathrm{T}-\mathrm{R}}=3$ using single and dual solvent formulations. Images (a) and (c) are optical and height mode AFM images respectively from a single solvent solution (mesitylene) while images (b) and (d) are from mesitylene-anisole dual solvent solution. Again, a substantial increase in the size of crystallite domains and decrease in surface roughness for the dual-solvent film is evident. The surface morphology is consistent with a 3-dimensional crystallization mode for the single solvent film as opposed to a 2-dimensional mode for the dual solvent film. 
formulations-Fig. 4a and b respectively. Fig. $4 \mathrm{c}$ and d are corresponding AFM scans from the same area. The images again clearly show that use of anisole as second solvent results in a dramatic decrease in surface roughness (from a typical root mean square roughness value of $20 \mathrm{~nm}$ for the single solvent to $2.5 \mathrm{~nm}$ for the dual solvent) and an increase in the size of crystalline domains of the films relative to films spin coated from a single solvent.

The surface of the mesitylene-anisole, $R_{\text {T-P }}=3$ film was characterized in greater detail by AFM (Fig. 5). In Fig. 5a, surface topography reveals a terraced morphology with stepped contours. This pattern is typical of a 2-dimensional growth mode indicating that during crystallization, molecular surface diffusion is high enough to promote a highly flat surface. In Fig. 5b, examination of the terraces using image cross-sections reveals that step heights correspond to an interlayer separation of $d \approx 1.6$ to $1.7 \mathrm{~nm}$ (steps $1,3,5$ ) or multiples of $d$ (steps 2, 4) as measured from the baseline (dashed segment); e.g. step 2 equals $3.4 \mathrm{~nm}$ corresponding to $3.4 \mathrm{~nm}=2 \times 1.7 \mathrm{~nm}$, i.e. two monolayers. From this cross-section, the average monolayer height was calculated to be $\langle d\rangle=1.65 \pm 0.08 \mathrm{~nm}$. A statistical analysis with more data from the same image is shown in the ESI†. If we consider that the dispersion around average is due to small measurement errors and other random sources of small errors, we can estimate an average monolayer height of $\langle d\rangle=1.64 \pm 0.04$ $\mathrm{nm}$. Both values are close to the $c$-axis unit cell $(1.68 \mathrm{~nm})$ of TIPS-pentacene. ${ }^{3}$

a)
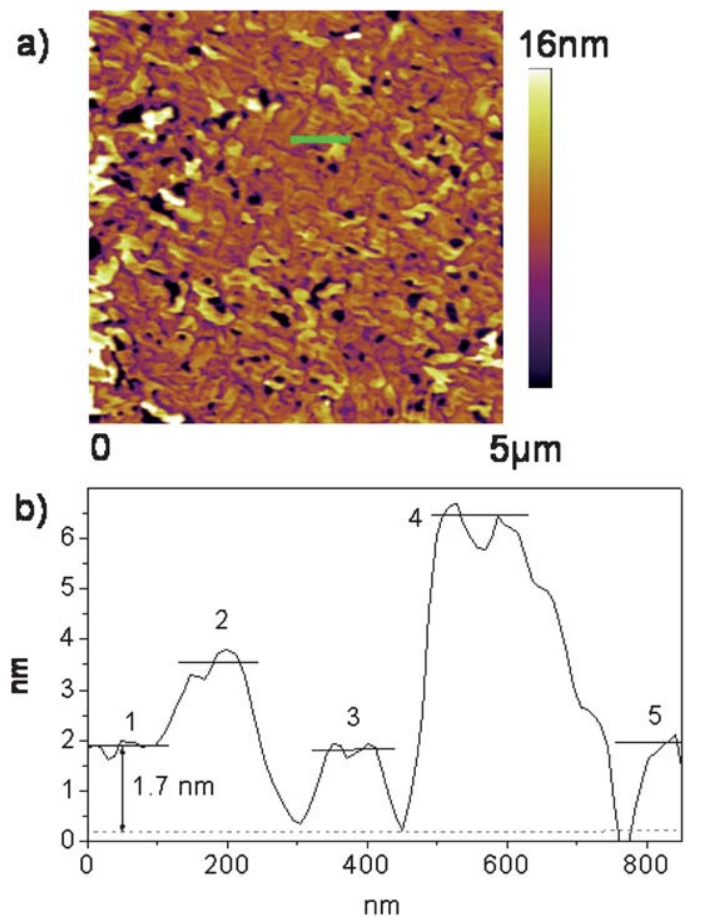

Fig. 5 (a) AFM surface topography (height mode) showing terraced morphology of typical TIPS-pentacene:PS film with $R_{\mathrm{T}-\mathrm{R}}=3$ deposited from mesitylene-anisole solution. (b) Cross-section along straight line segment marked green in image (a). Step heights correspond to multiples of a monolayer with height $\sim 1.6$ to $1.7 \mathrm{~nm}$ ( $c$-lattice parameter of TIPSpentacene is $1.68 \mathrm{~nm}$ ).

\section{Discussion}

\subsection{Phase-separation during deposition}

In the single solvent film, large elevations on the surface produced by elongated structures, consisting of needle-shaped crystals are clearly visible (Fig. 4c). Such structures are clearly absent in the anisole-mesitylene layers. The transition observed from needle-shaped crystallites (single solvent) to platelet-shaped crystallites (dual-solvent) is consistent with the previous work on the TIPS-pentacene morphology. ${ }^{14}$ Acene crystals are known to tend to aggregate along $\pi-\pi$ stacking directions and assume an "edge-on" orientation on various substrates, including glass. In ref. 14, the $\pi-\pi$ stacking [210] direction was identified as a pathway for high charge carrier transport which coincides with the long axis of needle-shaped TIPS-pentacene films. However, needle-shaped crystallites can be oriented at various angles not necessarily forming a continuous path from source to drain. Promoting platelet-shaped growth allows for better bridging of the electrodes and provides charge carrier pathways along another two directions [120] and [120], maximizing thus charge transport across the channel. As will be later discussed, this is achieved by improving diffusivity of the small molecule during crystallization via plasticization of the polymer matrix.

The terrace morphology of typical TIPS-pentacene:PS film with $R_{\mathrm{T}-\mathrm{R}}=3$ mesitylene-anisole solution showed step heights that are identical to the $c$-axis $(1.68 \mathrm{~nm})$ of TIPS-pentacene. This is a strong indication that the blend phase-separates during deposition and the small molecule segregates and crystallizes at the free surface. We here hypothesize that the improved mobilities of the higher TIPS-pentacene loaded films $\left(R_{\mathrm{T}-\mathrm{P}}=3\right)$ are due to a solid solution of the polymer with the remaining non-crystallized small molecules in the underlying layer.

\subsection{Methodology for selecting suitable solvent candidates}

We now turn our discussion to the selection process for suitable solvent candidates and to the mechanism that is involved in crystallization control. As indicated earlier, our approach consists of using a mixture of two solvents each of which is a better solvent of a different solid molecule. Initially, in order to find potential solvent candidates, we followed a theoretical assessment based on solubility parameter analysis. After this analysis, solubilities were experimentally tested and compared with theory.

Basic solubility theory states that a chemical (1) will be a good solvent for a specific polymer (2) if they have similar Hildebrand solubility parameters. ${ }^{15}$ In addition, for polar solutes, a further condition is required: good solubility is expected if solvent and solute have similar value of all three Hansen solubility parameters. According to the Hansen analysis, each chemical is assigned a dispersion force $\left(\delta_{\mathrm{d}}\right)$, polar interaction $\left(\delta_{\mathrm{p}}\right)$ and hydrogen-bonding $\left(\delta_{\mathrm{h}}\right)$ parameter ${ }^{16}$ The solid will be probably soluble in a solvent if the distance of the solvent from the center of the polymer solubility sphere, $R$, is less than the radius of interaction of the polymer $R_{\mathrm{o}}$.

$$
\sqrt{4\left(\delta_{\mathrm{d} 1}-\delta_{\mathrm{d} 2}\right)^{2}+\left(\delta_{\mathrm{p} 1}-\delta_{\mathrm{p} 2}\right)^{2}+\left(\delta_{\mathrm{h} 1}-\delta_{\mathrm{h} 2}\right)^{2}}=R<R_{\mathrm{o}}
$$

For PS, $R_{\mathrm{o}}$ is quite large ( $\left.\sim 12.7\right)$ as expected for a polymer that easily dissolves in a large range of solvents. The solubility parameters for the solvents and PS can be obtained from the 
literature. ${ }^{15,17}$ The solubility parameters for TIPS-pentacene, which has a smaller solubility sphere, were established from solubility tests. A fractional solubility plot of the compounds examined can be seen in Fig. 6. As expected, TIPS-pentacene whose solubilizing groups are alkyl compounds has a large $\delta_{\mathrm{d}}$ and small $\delta_{\mathrm{p}}$ and $\delta_{\mathrm{h}}$ parameters. Therefore it lies closer to the lower right-hand part of the solubility plot where aliphatics/ aromatics and alkyl aromatics such as mesitylene are commonly found. On the other hand, PS has $\delta_{\mathrm{p}}$ and $\delta_{\mathrm{h}}$ parameters larger than TIPS-pentacene and its position in the plot will be closer to aromatic ether and esters. This approach was used to identify possible favorable candidate solvents of each solid molecule. Nevertheless, one of the limitations of the solubility parameter approach is that some of the solvents with similar solubility parameters to the solutes will not dissolve as well as predicted. For this reason, we backed the expected solubilities with our own solubility tests. From such tests it was established that the solubility of PS in mesitylene is $\sim 0.3 \mathrm{~g} \mathrm{ml}^{-1}$. From the second solvents only 4-methylanisole, butyl acetate and anisole had solubility higher than that of mesitylene with anisole having the highest $\left(>0.4 \mathrm{~g} \mathrm{ml}^{-1}\right)$. On the other hand, solubility of TIPSpentacene in anisole was $\sim 1.5 \% \mathrm{w} / \mathrm{w}$ but was $>2 \% \mathrm{w} / \mathrm{w}$ in mesitylene whereas its solubility in 4-methylanisole was between that of mesitylene and anisole. In this way, the solubility series of TIPS-pentacene in those solvents with higher PS dissolving power than mesitylene was established as: mesitylene $>$ 4-methylanisole $>$ anisole $>$ butylacetate.

Since an initial stage of phase separation in the drying film would involve some type of molecular rotation or migration in a polymer environment, solvent molecules should provide a three-fold function in the crystallization process. They should not only serve as a medium for adequate solubilization but should also allow a timely, but not too fast, crystallization. Additionally, they should plasticize the polymer chain enough so

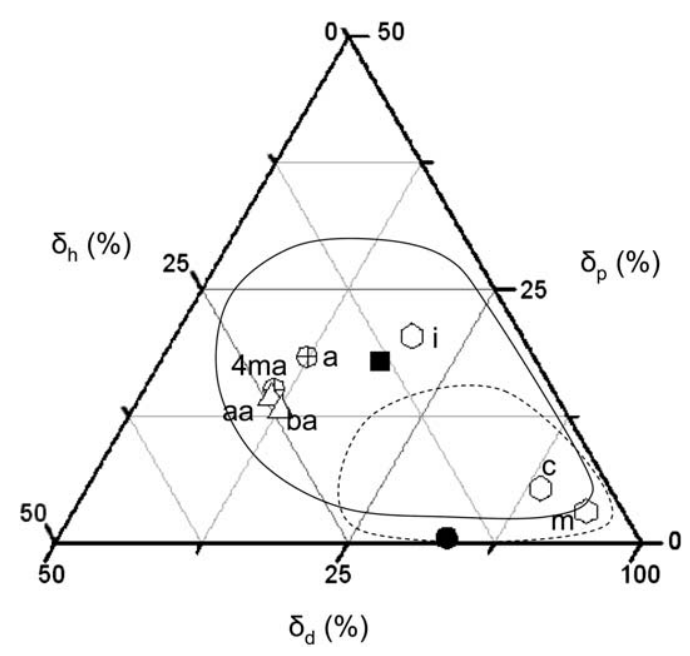

Fig. 6 Solubility plot (Teas graph) indicating fractional solubility

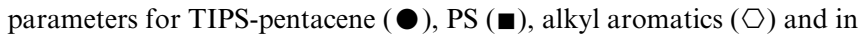
particular mesitylene (m), cumene (c), indane (i), aromatic esters $(\triangle)$ and in particular butyl acetate (ba), amyl acetate (aa) and finally aromatic ethers $(\oplus)$, anisole (a), 4-methylanisole (4ma). Contours are guides to the eye approximately indicating areas of high solubility for TIPS-pentacene (---) and PS (-). as to make molecular motion and migration possible. This would be critical at the early stages of phase separation. According to the above solubility series, anisole is highly compatible with the PS molecule but is not a very good TIPS-pentacene solvent. On the other hand mesitylene is a very good TIPS-pentacene solvent. Therefore, we attribute the improved morphology achieved for the mesitylene-anisole mixture to a plasticization effect of anisole on the PS molecule. The smaller crystal size observed in films from the single solvent solution can be partially attributed to a lower polymer chain mobility for this solvent environment which restricts the small molecule migration in the crystallization process. According to this mechanism, a morphology transition from needle-shaped (single solvent) to platelet-shaped (dual solvent) would be expected because the solvent would improve the crystallization kinetics during phase separation. The suboptimal performance of butyl acetate as second solvent could be attributed to the very poor solubility towards the TIPS-pentacene molecule, possibly resulting in premature crystallization at a very early stage of phase separation. Therefore, while we would expect that in an optimum solvent mixture, the main solvent dissolves the small molecule better while the second dissolves the polymer better than the small molecule, for optimum performance we would expect that the differentials between solubilities would also be important.

From the above discussion, we conclude that the present solubility parameter approach to selecting solvents is promising as it can explain in a qualitative way trends in the experiments we performed, whilst it led us to devices with record mobilities for a solution processed TIPS-pentacene based layer. It should be pointed out that further refinements of the present approach of utilizing solubility differences are needed. Due to the complexity of the dual solvent (quaternary system), further experiments with more mixing ratios varied over a broad range of combinations will be useful for further understanding and performance improvement. Additionally, the dual solvent approach should be considered in combination with optimisation of the $R_{\mathrm{T}-\mathrm{P}}$ ratio.

The possibility of exploring the polarity of solvent molecules as a means to control the final crystal morphology in organic TFTs has been raised before. Examples include the solvent vapor annealing approach ${ }^{\mathbf{1 8}, 19}$ and the solvent-assisted re-annealing of the active layer for semiconductor copolymers. ${ }^{20}$ Although it is possible that vapor based techniques could offer a route for device performance improvement for various molecules, including TIPS-pentacene, these are post-deposition procedures and as such are less versatile and more costly than the approach shown here.

Recently, solution, non-binder based techniques for TIPSpentacene have been explored which focus on optimizing deposition conditions in an effort to exercise control over crystalline morphology. ${ }^{21,22}$ These techniques achieve much lower mobilities than the one reported here. In addition, the nature of morphology control we have presented derives from optimization of the solvent mixture as opposed to environment controlling techniques. This allows for a larger processability window and adaptability to a variety of coating and printing deposition techniques, and thus much lower production costs. In addition, the demonstrated approach opens the possibility to fully use the advantages offered by blends in tuning rheological properties to suit specific deposition requirements without loss in device performance. 


\section{Conclusions}

In conclusion, a two-solvent blend processing approach is explored as a route to improved semiconductor device performance for small-molecule polymer blends. This approach combines the benefit of a relatively high boiling point solvent (mesitylene) with good acene solubility with the use of a second solvent (here anisole or 4-methylanisole) exhibiting higher polymer solubility. Solubility parameter analysis can be used as a first stage in identifying potential candidate solvents. Appropriately chosen solubility differentials of the two solvents enhance phase separation during deposition and lead to formation of larger crystallites and a flatter active surface in the device channel. In this way, we have achieved very high device mobilities. We have also shown that polydisperse amorphous polystyrene does not by itself limit device performance but under the right processing conditions the maximum potential of the small molecule can be exploited. Overall, the two-solvent blend approach allows control of the deposition process from within the solution as opposed to post-deposition or environment optimization techniques. It thus allows the use of a large window of deposition parameters offering a novel, versatile route to in-air and low cost fabrication of electronic devices.

\section{Experimental}

Amorphous, polydisperse polystyrene (PS), $M_{\mathrm{n}}=\sim 170000, M_{\mathrm{w}}$ $=\sim 350$ 000, was obtained from Sigma-Aldrich. Reagent grade solvents were obtained from Sigma or Merck [corresponding CAS number in brackets]: $n$-butyl acetate [123-86-4], $n$-amyl acetate [628-63-7], cumene (isopropylbenzene) [98-82-8], anisole (methoxybenzene) [100-66-3], mesitylene (1,3,5-trimethylbenzene) [108-67-8], 4-methylanisole [104-93-8] and indane (benzocyclopentane) [496-11-7].

Solubilities of TIPS-pentacene and PS were determined by mixing the two solids at ratios close to the solubility limit and stirring the solutions for several hours at room temperature. Then the solutions were left to settle for an idle period. If no undissolved particles were observed, the mixing and stirring process cycle was repeated until precipitation was obvious.

OSC formulations were obtained by first preparing separate $2 \%(\mathrm{w} / \mathrm{w})$ stock solutions of TIPS-pentacene in mesitylene as well as PS in a mesitylene or another solvent and stirring for $\sim 1$ day. The stock solutions were subsequently mixed in $1: 1$ or $3: 1(\mathrm{v} / \mathrm{v})$ ratios (referred to as $R_{\mathrm{T}-\mathrm{P}}=1$ and $R_{\mathrm{T}-\mathrm{P}}=3$ respectively) to yield the final formulation which was stirred for several hours. In this way, formulations constituted either a ternary, single solvent solution (two solutes and one solvent) or a quaternary, dual solvent solution (two solutes and two solvents).

The TFTs were fabricated with a top gate on Corning glass substrates. Top gate structures were used, as opposed to bottom gate structures, because resultant devices gave consistently higher mobilities presumably due to the tendency of the small molecule to segregate predominantly towards the free film surface under our deposition conditions. Following cleaning of the substrates by sonication in methanol and UV irradiation or oxygen plasma ashing, source-drain electrodes $\sim 40 \mathrm{~nm}$ thick were thermally evaporated from Au via a shadow mask, yielding $20 \mu \mathrm{m}$ length and $1000 \mu \mathrm{m}$ width channels. The surface was then treated by spin coating an $\sim 10 \mathrm{mmol} \mathrm{L}^{-1}$ solution of pentafluorothiophenol in isopropanol, followed by the application of the OSC formulation also by spin coating (2000 rpm for $60 \mathrm{~s}$ ). The layer was then dried at $100{ }^{\circ} \mathrm{C}$ for $1 \mathrm{~min}$ in air. The dielectric (amorphous fluoropolymer, CYTOP, Asahi Glass) was subsequently spun onto the $\mathrm{OSC}$ and dried at $100{ }^{\circ} \mathrm{C}$ for $2 \mathrm{~min}$ in air to yield a $1 \mu \mathrm{m}$ thick dielectric layer, as confirmed by profilometry. CYTOP was preferred as it offers printable potential, low curing point, good orthogonality and good encapsulating properties due to its high hydrophobicity and, finally, very smooth films (typical rms roughness is in the single-digit nanometre regime). The geometric capacitance of the dielectric layer was measured to be $C=1.7 \mathrm{nF} \mathrm{cm}{ }^{-2}$. Finally, $\sim 40 \mathrm{~nm} \mathrm{Au} \mathrm{gate} \mathrm{electrodes} \mathrm{were}$ evaporated using a shadow mask. All devices were fabricated in air using identical fabrication protocol conditions and had identical electrodes with channel length, $L=20 \mu \mathrm{m}$ and channel width, $W=1000 \mu \mathrm{m}$.

Electrical characterization of the TFT devices was performed in air using an Agilent 4155C semiconductor parameter analyzer. Linear $\left(\mu_{\text {lin }}\right)$ and saturation $\left(\mu_{\text {sat }}\right)$ device mobilities were extracted from transfer curves based on the standard, gradual channel approximation model:

$$
\mu_{\text {lin }}=\frac{L}{C W} \frac{1}{V_{\mathrm{D}}} \frac{\partial I_{\mathrm{D}}}{\partial V_{\mathrm{G}}} \text { and } \mu_{\mathrm{sat}}=\frac{2 L}{W C_{i}}\left(\frac{\partial \sqrt{I_{\mathrm{DS}}}}{\partial V_{\mathrm{G}}}\right)^{2}
$$

where $L$ and $W$ are respectively the device channel length and width, $V_{\mathrm{G}}$ and $V_{\mathrm{D}}$ are respectively the gate and drain voltages, $I_{\mathrm{D}}$ is the drain current and $C$ is the dielectric geometric capacitance as given above.

Optical microscopy was carried out using an Nikon Eclipse LV-100 under cross-polarizers. AFM surface scans were performed using an MFP-3D Asylum instrument in tapping (noncontact) mode.

\section{Acknowledgements}

Thanks are due to Dr Philipp Thurner, School of Engineering Sciences, University of Southampton for providing access to an AFM microscope and for useful suggestions and discussions. Thanks are due to all Merck staff at Chilworth for suggestions and discussions. This work was supported by Knowledge Transfer Partnership Program 1326.

\section{References}

1 A. C. Arias, J. D. MacKenzie, I. McCulloch, J. Rivnay and A. Salleo, Chem. Rev., 2010, 110, 3.

2 J. E. Anthony, D. L. Eaton and S. R. Parkin, Org. Lett., 2002, 4, 15. 3 S. K. Park, T. N. Jackson, J. E. Anthony and D. A. Mourey, Appl. Phys. Lett., 2007, 91, 063514.

4 D. H. Kim, D. Y. Lee, H. S. Lee, W. H. Lee, Y. H. Kim, J. I. Han and K. Cho, Adv. Mater., 2007, 19, 678.

5 P.-T. Wu, H. Xin, F. S. Kim, G. Ren and S. A. Jenekhe, Macromolecules, 2009, 42, 8817.

6 B. A. Brown, J. Veres, R. M. Anemian, R. Williams, S. D. Ogier and S. W. Leeming, WO Pat., 2005/055248, 2005; B. A. Brown, J. Veres, R. M. Anemian, R. Williams, S. D. Ogier and S. W. Leeming, US Pat., 7576208 B2, 2009.

7 T. Ohe, M. Kuribayashi, R. Yasuda, A. Tsuboi, K. Nomoto, K. Satori, M. Itabashi and J. Kasahara, Appl. Phys. Lett., 2008, 93, 053303. 
8 R. Hamilton, J. Smith, S. Ogier, M. Heeney, J. E. Anthony, I. McCulloch, J. Veres, D. D. C. Bradley and T. D. Anthopoulos, Adv. Mater., 2009, 21, 1166.

9 J. Kang, N. Shin, D. Y. Jang, V. M. Prabhu and D. Y. Yoon, J. Am. Chem. Soc., 2008, 130, 12273.

10 J. Smith, R. Hamilton, I. McCulloch, N. Stingelin-Stutzmann, M. Heeney, D. D. C. Bradley and T. D. Anthopoulos, J. Mater. Chem., 2010, 20, 2562.

11 M.-B. Madec, D. Crouch, G. R. Llorente, T. J. Whittle, M. Geoghegan and S. G. Yeates, J. Mater. Chem., 2008, 18, 3230 .

12 J.-H. Kwon, S.-I. Shin, K.-H. Kim, M. J. Cho, K. N. Kim, D. H. Choi and B.-K. Ju, Appl. Phys. Lett., 2009, 94, 013506.

13 C. S. Kim, S. Lee, E. D. Gomez, J. E. Anthony and Y. L. Loo, Appl. Phys. Lett., 2008, 93, 103302.

14 J. Chen, C. K. Tee, M. Shtein, D. C. Martin and J. Anthony, Org. Electron., 2009, 10, 696.
15 A. F. M. Barton, Handbook of Solubility Parameters and Other Cohesion Parameters, CRC Press, 2nd edn, 1991.

16 C. Hansen, Hansen Solubility Parameters: A User's Handbook, CRC press, 2nd edn, 2007.

17 C. Hansen, Hansen Solubility Parameters Software, HSPiP, 3rd edn.

18 G. De Luca, T. E. Treossi, A. Liscio, J. M. Mativetsky, L. M. Scolaro, V. Palermo and P. Samori, J. Mater. Chem., 2010, 20, 2493.

19 K. C. Dickey, J. E. Anthony and Y.-L. Loo, Adv. Mater., 2006, 18, 1721.

20 C. Di, K. Lu, L. Zhang, Y. Liu, Y. Guo, X. Sun, Y. Wen, G. Yu and D. Zhu, Adv. Mater., 2010, 22, 1273.

21 X. Li, B. K. C. Kjellander, J. E. Anthony, C. W. M. Bastiaansen, D. J. Broer and G. H. Gelinck, Adv. Funct. Mater., 2009, 19, 3610.

22 C. W. Sele, B. K. C. Kjellander, B. Niesen, M. J. Thornton, J. B. P. H. van der Putten, K. Myny, H. J. Wondergem, A. Moser, R. Resel, A. J. J. M. van Breemen, N. van Aerle, P. Heremans, J. E. Anthony and G. H. Gelinck, Adv. Mater., 2009, 21, 4926. 